\title{
Riesgo de deterioro cognitivo en personas mayores de las subregiones de Antioquia, Colombia*
}

\author{
Angela Segura Cardona** \\ Maria Garzón Duque ${ }^{\star \star \star}$ \\ Doris Cardona Arango ${ }^{\star \star \star \star}$ \\ Alejandra Segura Cardona ${ }^{\star \star \star \star \star}$
}

En este artículo, se buscó conocer la prevalencia de riesgo de deterioro cognitivo y su relación con factores demográficos, sociales y funcionales en las personas mayores de las subregiones de Antioquia, Colombia. Se trata de un estudio cuantitativo, transversal analítico, con fuente de información primaria. La muestra fue probabilística y se determinó el riesgo de deterioro cognitivo con la escala minimental. Así se encontró que el 83,1\% de los mayores presenta algún riesgo de deterioro cognitivo, principalmente las mujeres, y está asociado significativamente con vivir en la subregión del Magdalena Medio y Medellín, tener mayor edad, no tener pareja, no tener escolaridad, tener escasos recursos sociales, no participar en grupos comunitarios, tener mala percepción de la calidad de vida, estar insatisfecho con la salud y tener dependencia funcional para realizar las actividades básicas. Estos resultados permiten orientar los programas de salud pública relacionados con la inclusión de las personas mayores hacia programas educativos, la participación en grupos comunitarios, la tamización constante en los servicios de salud, el fortalecimiento de los recursos sociales y el fomento de la actividad física en la vejez, para lograr un envejecimiento saludable con mejor calidad de vida.

Palabras clave: Adulto mayor. Cognición. Envejecimiento.

\footnotetext{
* Las investigadoras agradecen a los adultos mayores encuestados, a las dependencias financiadoras, auxiliares, estudiantes y encuestadores que hicieron posible la realización de este proyecto. Este proyecto de investigación fue ejecutado a través de un convenio de asociación entre el Departamento de Antioquia, Secretaría Seccional de Salud y Protección Social de Antioquia y la Universidad CES, Facultad de Medicina (2012cf164156).

** Universidad CES, Medellín, Antioquia, Colombia (asegura@ces.edu.co).

${ }^{\star * \star}$ Universidad CES, Medellín, Antioquia, Colombia (mgarzon@ces.edu.co).

**** Universidad CES, Medellín, Antioquia, Colombia (dcardona@ces.edu.co).

***** Universidad CES, Medellín, Antioquia, Colombia (alejasegura10@gmail.com).
} 


\section{Introducción}

El crecimiento de la población de mayor edad está teniendo un comportamiento más acelerado que los demás grupos de edad, situación que lleva a un rápido envejecimiento poblacional (OMS, 2002). Se observa un aumento en el número de personas adultas mayores que padecen enfermedades mentales, en gran medida relacionadas con la edad, como demencia y depresión (FEDERACIÓN MUNDIAL DE SALUD MENTAL, 1999).

En la población adulta mayor se observa además un deterioro en los procesos psicológicos superiores, que implican la capacidad de atención (SANCHÉZ; PERÉZ, 2008), percepción, inteligencia, memoria de trabajo, praxias y funciones ejecutivas. El deterioro mental tiende a agudizarse por la dificultad de detección oportuna (AGRIGOROAEI; LACHMAN, 2011).

En la vejez pueden presentarse una disminución en la precisión y observación de señales y un aumento en la dificultad para discriminar estímulos relevantes e irrelevantes y en la capacidad para atender a dos estímulos simultáneamente (VIVAS; ANDRÉS; CANET, 2011). También puede verse afectada la percepción, ${ }^{1}$ principalmente por el deterioro de los órganos sensoriales que comienzan a no percibir adecuadamente los estímulos provenientes del entorno. La inteligencia cristalizada (capacidades, estrategias y conocimientos), que representa el nivel de desarrollo cognitivo alcanzado, se consolida con la edad y no sufre un declive importante con los años, mientras que la inteligencia fluida (capacidad para adaptarse y afrontar situaciones) implica la puesta en marcha de las funciones ejecutivas para generar respuestas alternativas ante situaciones novedosas presenta un declive en la vejez de acuerdo a los recursos con que cuenta cada persona (VIVAS; ANDRÉS; CANET, 2011).

En términos generales, la capacidad de sostener información en la memoria inmediata se mantiene en la vejez. Sin embargo, la capacidad de mantener presentes múltiples datos y manipularlos mentalmente disminuye en cierta medida conforme aumenta la edad (VIVAS; ANDRÉS; CANET, 2011). Algunos trastornos de depresión, de atención, del lenguaje, visuales y auditivos interfieren en el buen funcionamiento de la memoria y en ocasiones pueden simular demencia (BIURRUMUNZUÉ, 2000). Según un estudio realizado en Cuba, estos problemas afectan a la mitad de las personas mayores de 65 años, mientras que la demencia es padecida por el 5\% (PÉREZ, 2005). Para evaluar la capacidad cognitiva de los adultos existen instrumentos de medición, rápidos, confiables y validados en el medio (IBARRA; VILLARREAL; SALINAS, 2006), principalmente el Mini Mental State Examination (MMSE) (FOLSTEIN; FOLSTEIN; HUGH, 1975).

La población mayor de sesenta años del departamento de Antioquia evidencia un aumento del 68,17\%, pasando de 313.328 en el año 1993 a 526.949 en 2005, con una tasa anual de crecimiento del 5,7\% (GOBERNACIÓN DE ANTIOQUIA, 2015). El presente estudio tenía como objetivo conocer la prevalencia de riesgo de deterioro cognitivo y su relación

\footnotetext{
${ }^{1}$ Se toma por percepción la capacidad de recibir, elaborar e interpretar información proveniente del entorno.
} 
con factores demográficos, sociales, económicos y funcionales, en personas mayores de Antioquia, Colombia.

\section{Materiales y métodos}

Se presenta aquí un estudio cuantitativo transversal observacional analítico con fuentes primarias de información para el que se capacitaron quince encuestadores para recoger la información, contratados por la Universidad CES de Medellín, Colombia. Para el cálculo de la muestra se tomaron como referencia 671.847 personas de sesenta años y más años que vivían en la zona urbana o en centros rurales poblados de Antioquia, según las proyecciones para el año 2012, calculadas por el Departamento Administrativo Nacional de Estadística (DANE, 2007). La muestra se calculó con la fórmula para poblaciones finitas: $95 \%$ de confianza, $5 \%$ como error máximo permitido, 1,0 como efecto de diseño (deff) y $50 \%$ como proporción de mayores con buen funcionamiento cognitivo. La muestra mínima calculada de 3.790 se amplió en un $10 \%$ para corregir posibles pérdidas de información y quedó finalmente en 4.248 adultos mayores, representativos de las nueve subregiones y de la capital del departamento, con los que se calcularon factores de expansión y los errores de muestreo.

La muestra se seleccionó mediante un muestreo probabilístico para que todos los adultos mayores de la subregión tuvieran la posibilidad de ser incluidos en el estudio. Se empleó un muestreo polietápico (en cuatro etapas), en el que inicialmente se seleccionaron cuatro municipios de manera aleatoria (unidad primaria de muestreo). Posteriormente se seleccionaron las manzanas, sectores o centros rurales poblados dentro de los municipios (unidad secundaria de muestreo) y finalmente se seleccionaron las viviendas (unidad terciaria de muestreo). Allí se encuestó a todas las personas de sesenta años y más (unidad final de muestreo y primera de análisis) (CARDONA; SEGURA; GARZÓN, 2013). En los municipios se tomaron entre 104 y 107 adultos mayores.

El cuestionario aplicado a los adultos mayores cuenta con escalas ya validadas. El grado de riesgo de deterioro cognitivo se midió a partir de la escala MMSE de Folstein, que se emplea para detectar grados de deterioro cognitivo en cada una de las funciones psicológicas superiores (CIOMS; OMS, 2002). Las variables independientes fueron tomadas según estudios previos, donde se encuentra que el deterioro está relacionado con condiciones demográficas (sexo, edad, área de procedencia y estado conyugal). Para la capacidad funcional se usaron el índice de Katz, que evalúa la capacidad de efectuar una serie de tareas básicas (KATZ et al., 1963), la escala de recursos sociales OARS para valorar el apoyo social recibido (LETURIA et al., 2001) y el Cuestionario General de Salud de Goldberg para evaluar salud percibida (GOLDBERG; HILLER, 1979).

Se realizó análisis univariado para caracterizar la población mayor y cada subregión, tanto de la variable dependiente (riesgo de deterioro cognitivo medido de forma dicotómica: con y sin riesgo) como de las variables independientes (estado conyugal, escolaridad, 
edad, calidad de vida, dependencia funcional y satisfacción con el estado de salud, que fueron recategorizadas). Con ellas se calcularon medidas de frecuencia medidas descriptivas, usando el factor de expansión como técnica de estadística inferencial, que se basó en el cálculo de ponderadores para reconstruir la población de adultos mayores del departamento de Antioquia, Colombia, ajustado por factores de corrección como no respuesta, cobertura, sexo, zona de procedencia, entre otros. El análisis univariado fue calculado con factores de expansión, para representar la totalidad de adultos mayores del departamento.

Posteriormente, se hizo análisis bivariado con los datos de la encuesta, para relacionar el riesgo de deterioro cognitivo con los aspectos estudiados, apoyado en el cálculo de la razón de prevalencias (RP) crudas, intervalo de confianza del 95\% (IC 95\%) y la prueba estadística chi cuadrado $\left(x^{2}\right)$, considerada asociación con nivel de significación menor del $5 \%$. Igualmente, con el uso de la regresión logística se realizó un análisis multivariado estratificado y una regresión logística explicativa para el cálculo del RP ajustado, donde se incluyeron todas las variables con significancia estadística $(p<0,05)$. Con este análisis se controlaron la confusión y la interacción de variables demográficas con cada una de las variables independientes.

Éticamente, este proyecto obtuvo aprobación de la Universidad CES y del Comité Institucional de Ética (Acta 58).

\section{Resultados}

\section{Perfil de las personas mayores de Antioquia}

Las personas mayores de Antioquia registraron una edad media de 70,42 años ( $\mathrm{S}=$ 8,27). Las mujeres tenían 70,31 años $(S=8,43)$, con edades que oscilaban entre 60 y 112 años, mientras que los hombres tenían setenta $(S=7,82)$, en un rango de 60 a 103 años. La mayor proporción de adultos mayores eran casados (38,3\%), seguidos de los viudos (33,4\%). Los adultos mayores casados se presentaron en mayor medida en Oriente, Suroeste, Valle de Aburrá y Occidente, y los viudos tuvieron mayor presencia en Medellín, Nordeste y Norte. El 72,7\% de los adultos mayores tenía como máximo nivel de educación primaria completa, pero un $14,8 \%$ no había alcanzado ningún nivel de instrucción. La mayoría de adultos mayores residía en el área urbana. El 81,7\% (522.966) de los adultos mayores refiere ser independiente funcional para realizar las actividades de la vida diaria.

\section{Riesgo de deterioro cognitivo}

En los resultados de las funciones mentales superiores evaluadas con el MMSE se identifica que el $16,8 \%$ (109.783) de los adultos mayores no presenta ningún riesgo de deterioro cognitivo según la escala de medición usada, ya que no se diagnostica el evento. Sin embargo, el 83,1\% (543.997) presentó algún grado de deterioro, el 46,8\% (306.507) presentó riesgo leve, el 32,1\% (209.888) moderado y el 4,2\% (27.602) riesgo severo de 
deterioro. Dicotomizada la variable de interés, se encuentra que la prevalencia de riesgo de deterioro es del 83,2\% (544.690) frente al 16,8\% sin este riesgo.

En la capacidad de orientación temporoespacial un alto porcentaje demostró adecuada orientación (75\%). En cuanto a la capacidad de memoria de fijación al nombrar tres palabras con intervalos de tiempo determinados, los adultos mayores tuvieron un alto porcentaje en la primera palabra (95,7\%), pero a medida que avanzaba la prueba se dificultaba el recuerdo de las demás. Los planteamientos empleados para medir la capacidad atencional y el cálculo se midieron con ejercicios como repetir en series de siete los números del cien hacia atrás o deletrear la palabra "mundo" al revés. Solo el $25 \%$ de los adultos mayores logró hacerlo en el primer intento y a medida que avanzaba la prueba este planteamiento se hacía más complejo para los adultos mayores, por lo que los porcentajes de respuesta disminuían cada vez más.

La capacidad de evocar información recibida previamente luego de un intervalo de tiempo mayor y una serie de estímulos externos diversos disminuyó la capacidad de memoria de evocación de los adultos mayores: un poco más de la mitad de ellos (56\%) logró recordar solo la primera palabra y el recuerdo de las demás iba disminuyendo en porcentaje gradual. La capacidad lingüística de los adultos mayores se midió mediante ejercicios que abarcaban lenguaje comprensivo, expresivo y escrito: la propiedad expresiva del lenguaje tuvo un porcentaje de $97,5 \%$. Por otro lado, la parte comprensiva obtuvo valores mayores del $80 \%$. Cabe resaltar que la capacidad lingüística más alterada en los adultos mayores fue la escritura, dado el porcentaje menor en comparación con las demás (59,7\%).

\section{Factores asociados al riesgo de deterioro cognitivo}

Las variables que conforman el factor demográfico están asociadas estadísticamente con el riesgo de deterioro cognitivo, exceptuando el área de procedencia y el sexo. Las personas mayores residentes en las subregiones de Magdalena Medio, Nordeste y en la ciudad de Medellín registraron una prevalencia de riesgo más alta, mientras los habitantes residentes en las subregiones de Occidente, Bajo Cauca y Oriente registraron la menor (Gráfico 1).

Se aumenta la probabilidad de padecer el riesgo cuando se vive en las subregiones de Magdalena Medio ( $R P=2,44[1,593-3,737])$, Norte $(R P=1,38)$, Suroeste $(R P=1,15)$ y Oriente $(R P=1,14)$ cuando se compara con los adultos residentes en la subregión del Valle de Aburra. Vivir en las subregiones de Occidente y Bajo Cauca disminuye esta probabilidad y solo es estadísticamente significativo en el Magdalena Medio (Tabla 1).

De los mayores que tenían riesgo de deterioro cognitivo, el 65,7\% tenía entre 60 y 74 años (adulto mayor joven), pero el aumento de la edad aumenta la probabilidad de padecerlo $(\mathrm{RP}=2,68$ [2,161-3,315]). El 61,9\% de las mujeres y el 38,1\% de los hombres mayores presentaron el riesgo, indicando que por un hombre en riesgo hay 0,99 mujeres en esta misma situación ( $R P=0,99)$. Asimismo, el 91,7\% de los que presentaron deterioro cognitivo vivía en la zona urbana y vivir en el área rural aumenta en un $19 \%$ dicho riesgo (Tabla 1). 
GRÁFICO 1

Prevalencia de riesgo de deterioro cognitivo en persona mayor, según subregión de residencia Departamento de Antioquia - 2012

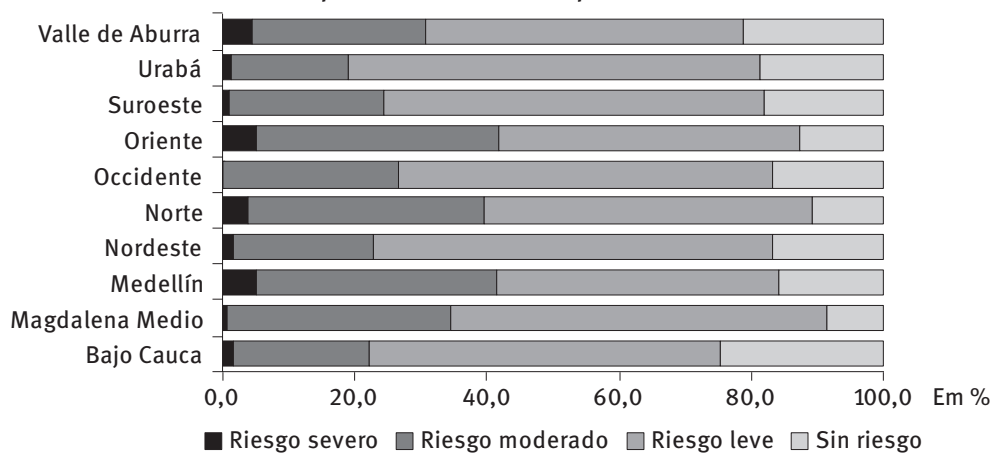

Fuente: Proyecto Situación de salud y condiciones de vida de los adultos mayores. Departamento de Antioquia, 2012.

TABLA 1

Riesgo de deterioro cognitivo en persona mayor asociado a factores demográficos Departamento de Antioquia, 2012

\begin{tabular}{|c|c|c|c|c|c|c|c|c|}
\hline \multirow{3}{*}{$\begin{array}{c}\text { Factores } \\
\text { demográficos }\end{array}$} & \multicolumn{4}{|c|}{ Deterioro cognitivo } & \multirow{3}{*}{$\begin{array}{c}\text { Pruęba } \\
x^{2}\end{array}$} & \multirow{3}{*}{$\begin{array}{l}\text { Valor } \\
\text { de p }\end{array}$} & \multirow{3}{*}{ RP } & \multirow{3}{*}{ IC $95 \%$} \\
\hline & \multicolumn{2}{|c|}{ Con riesgo } & \multicolumn{2}{|c|}{ Sin riesgo } & & & & \\
\hline & $\mathrm{N}$ & $\%$ & $\mathrm{~N}$ & $\%$ & & & & \\
\hline \multicolumn{9}{|l|}{ Subregión } \\
\hline Bajo Cauca & 337 & 9,5 & 96 & 13,7 & \multirow{10}{*}{38,863} & \multirow{10}{*}{$0,000^{*}$} & 0,77 & $0,544-1,078$ \\
\hline Magdalena Medio & 414 & 11,7 & 36 & 5,1 & & & 2,44 & $1,597-3,736$ \\
\hline Medellín & 395 & 11,1 & 80 & 11,4 & & & 1,05 & $0,739-1,488$ \\
\hline Nordeste & 363 & 10,2 & 71 & 10,1 & & & 1,08 & $0,756-1,556$ \\
\hline Norte & 351 & 9,9 & 54 & 7,7 & & & 1,38 & $0,940-2,025$ \\
\hline Occidente & 308 & 8,7 & 91 & 12,9 & & & 0,85 & $0,595-1,206$ \\
\hline Oriente & 337 & 9,5 & 63 & 9,0 & & & 1,21 & $0,828-1,750$ \\
\hline Suroeste & 357 & 10,1 & 66 & 9,4 & & & 1,15 & $0,797-1,656$ \\
\hline Urabá & 344 & 9,7 & 74 & 10,5 & & & 1,00 & $0,701-1,436$ \\
\hline Valle de Aburrá & 339 & 9,6 & 72 & 10,2 & & & 1,00 & - \\
\hline \multicolumn{9}{|l|}{ Persona mayor } \\
\hline 60 a 74 años & 2.340 & 65,7 & 574 & 83,7 & \multirow{2}{*}{81,331} & \multirow{2}{*}{$0,000^{*}$} & 1,00 & - \\
\hline $75+$ años & 1.222 & 34,3 & 112 & 16,3 & & & 2,68 & $2,161-3,315$ \\
\hline \multicolumn{9}{|l|}{ Sexo } \\
\hline Hombre & 1.357 & 38,1 & 259 & 37.8 & \multirow[t]{2}{*}{0,028} & \multirow[t]{2}{*}{0,866} & 1,00 & - \\
\hline Mujer & 2.205 & 61,9 & 427 & 62,2 & & & 0,99 & $0,833-1,167$ \\
\hline \multicolumn{9}{|l|}{ Estado conyugal } \\
\hline Sin pareja & 1.978 & 55,5 & 324 & 47,2 & \multirow{2}{*}{15.877} & \multirow{2}{*}{$0,000^{*}$} & 1,40 & $1,184-1,644$ \\
\hline Con pareja & 1.584 & 44,5 & 362 & 52,8 & & & 1,00 & - \\
\hline \multicolumn{9}{|l|}{ Escolaridad } \\
\hline Sin educación & 937 & 26,3 & 57 & 8,3 & \multirow{4}{*}{220,333} & \multirow{4}{*}{$0,000^{*}$} & 9,03 & $6,602-12,360$ \\
\hline Primaria & 2.221 & 62,4 & 407 & 59,3 & & & 2,99 & $2,468-3,644$ \\
\hline Secundaria & 404 & 11,3 & 222 & 32,4 & & & 1,00 & - \\
\hline \multicolumn{7}{|l|}{ Área de residencia } & & \\
\hline Urbana & 3.202 & 91,7 & 629 & 92,9 & \multirow[t]{2}{*}{1,120} & \multirow[t]{2}{*}{0,290} & 1,00 & - \\
\hline Rural & 290 & 8,3 & 48 & 7,1 & & & 1,19 & $0,864-1,630$ \\
\hline
\end{tabular}

Fuente: Proyecto Situación de salud y condiciones de vida de los adultos mayores. Departamento de Antioquia, 2012.

*Asociación estadísticamente significativa con $\mathrm{p}<0,05$. 
Los adultos sin pareja (solteros, separados o viudos) tienen más probabilidad de deterioro en un $40 \%$ con respecto a aquellos que tienen pareja (casados y en unión libre). Los mayores sin ningún nivel educativo o que solo alcanzaron la primaria, tienen mayor probabilidad de deterioro cognitivo (aproximadamente un 88,7\%) en comparación con aquellos adultos que alcanzaron educación secundaria. ( $\mathrm{RP}=9,03$ [6,602; 12,360]). (Tabla 1).

De los adultos mayores con riesgo de deterioro cognitivo, el $76,8 \%$ tenía recursos sociales deteriorados, ${ }^{2}$ situación que aumenta la probabilidad de padecer el riesgo en un $61 \%(R P=1,61[1,334-1,941])$. Asimismo, el 63,3\% no participaba en actividades grupales comunitarias, lo que implica un aumento de la probabilidad de deterioro $(\mathrm{RP}=1,35)$. El $15,7 \%$ fuma en la actualidad y el 10,9\% consume bebidas alcohólicas. Se encontró que el consumo de bebidas alcoholicas reduce la probabilidad de padecer el riesgo de deterioro en un $41 \%(R P=0,59[0,469-0,734])$. Otros aspectos sociales que aumentan el riesgo son percibir una mala calidad de vida $(R P=1,55)$ y estar insatisfecho con el estado de salud $(\mathrm{RP}=1,90)$. El 19,3\% de los adultos mayores con riesgo de deterioro cognitivo manifestó tener alguna dependencia funcional en la realización de las actividades básicas, aumentando su riesgo en un $61 \%$ (Tabla 2 ).

TABLA 2

Riesgo de deterioro cognitivo en persona mayor asociado a factores sociales Departamento de Antioquia - 2012

\begin{tabular}{|c|c|c|c|c|c|c|c|c|}
\hline \multirow{3}{*}{ Factores sociales } & \multicolumn{4}{|c|}{ Deterioro cognitivo } & \multirow{3}{*}{$\begin{array}{c}\text { Prueba } \\
x^{2}\end{array}$} & \multirow{3}{*}{$\begin{array}{l}\text { Valor } \\
\text { de p }\end{array}$} & \multirow{3}{*}{ RP } & \multirow{3}{*}{ IC $95 \%$} \\
\hline & \multicolumn{2}{|c|}{ Con riesgo } & \multicolumn{2}{|c|}{ Sin riesgo } & & & & \\
\hline & $\mathbf{N}$ & $\%$ & $\mathrm{~N}$ & $\%$ & & & & \\
\hline \multicolumn{9}{|l|}{ Recursos sociales } \\
\hline Buenos & 723 & 23,2 & 202 & 32,7 & 24,692 & $0,000^{*}$ & 1,00 & - \\
\hline Deteriorados & 2.390 & 76,8 & 415 & 67,3 & & & 1,61 & 1,334-1,941 \\
\hline \multicolumn{9}{|l|}{ Afiliación al SGSSS } \\
\hline $\mathrm{Si}$ & 3.411 & 95,8 & 668 & 97,4 & 3,853 & 0,050 * & 1,00 & - \\
\hline No & 151 & 4,2 & 18 & 2,6 & & & 1,64 & $1,001-2,697$ \\
\hline \multicolumn{9}{|l|}{ Régimen de afiliación } \\
\hline Contributivo & 1.065 & 29,9 & 298 & 43,4 & 47,734 & $0,000^{*}$ & 1,00 & - \\
\hline Subsidiado & 2.314 & 65,0 & 362 & 52,8 & & & 1,79 & $1,510-2,119$ \\
\hline Vinc/ninguno & 183 & 5,1 & 26 & 3,8 & & & 1,97 & $1,281-3,029$ \\
\hline \multicolumn{9}{|l|}{ Participación en grupos } \\
\hline Participa & 1.307 & 36,7 & 301 & 43,9 & 12,566 & $0,000^{*}$ & 1,00 & - \\
\hline No participa & 2.255 & 63,3 & 385 & 56,1 & & & 1,35 & $1,143-1,592$ \\
\hline \multicolumn{9}{|l|}{ Consumo de cigarrillo } \\
\hline $\mathrm{Si}$ & 559 & 15,7 & 79 & 11,5 & 7,796 & $0,005^{\star}$ & 1,43 & $1,113-1,839$ \\
\hline No & 3.003 & 84,3 & 607 & 88,5 & & & 1,00 & - \\
\hline \multicolumn{9}{|l|}{ Consumo de alcohol } \\
\hline $\mathrm{Si}$ & 387 & 10,9 & 118 & 17,2 & 21,646 & $0,000^{*}$ & 0,59 & $0,469-0,734$ \\
\hline No & 3.175 & 89,1 & 568 & 82,8 & & & 1,00 & - \\
\hline \multicolumn{9}{|l|}{ Calidad de vida } \\
\hline Mala & 2.062 & 57,9 & 322 & 46,9 & 27,742 & $0,000^{*}$ & 1,55 & 1,319-1,831 \\
\hline Buena & 1.500 & 42,1 & 364 & 53,1 & & & 1,00 & - \\
\hline
\end{tabular}

\footnotetext{
$\overline{2}$ Relaciones sociales no satisfactorias, poca familia y pocos amigos.
} 


\begin{tabular}{|c|c|c|c|c|c|c|c|c|}
\hline \multirow{3}{*}{ Factores sociales } & \multicolumn{4}{|c|}{ Deterioro cognitivo } & \multirow{3}{*}{$\begin{array}{c}\text { Prueba } \\
x^{2}\end{array}$} & \multirow{3}{*}{$\begin{array}{l}\text { Valor } \\
\text { de } p\end{array}$} & \multirow{3}{*}{$\mathrm{RP}$} & \multirow{3}{*}{ IC $95 \%$} \\
\hline & \multicolumn{2}{|c|}{ Con riesgo } & \multicolumn{2}{|c|}{ Sin riesgo } & & & & \\
\hline & $\mathrm{N}$ & $\%$ & $\mathrm{~N}$ & $\%$ & & & & \\
\hline \multicolumn{9}{|c|}{ Satisfacción con la salud } \\
\hline Insatisfecho & 1.194 & 33,5 & 144 & 21,0 & 40,850 & $0,000^{*}$ & 1,90 & $1,559-2,310$ \\
\hline Satisfecho & 2.368 & 66,5 & 542 & 79,0 & & & 1,00 & - \\
\hline \multicolumn{9}{|l|}{ Capacidad funcional } \\
\hline Dependencia & 688 & 19,3 & 89 & 13,0 & 19,246 & $0,000^{\star}$ & 1,61 & $1,266-2,037$ \\
\hline Independientes & 2.874 & 80,7 & 597 & 87,0 & & & 1,00 & - \\
\hline
\end{tabular}

Fuente: Proyecto Situación de salud y condiciones de vida de los adultos mayores. Departamento de Antioquia, 2012.

*Asociación estadísticamente significativa con $\mathrm{p}<0,05$.

Factores que más explican la presencia de riesgo de deterioro cognitivo

Con el fin de controlar el efecto confusor que pudieran generar algunas variables sobre la relación con el evento, se procedió a construir un modelo multivariado donde se incluyeran todas las variables con significación estadística $(p<0,05)$ y así determinar la fuerza de la asociación (RP) ajustada por las demás variables.

La probabilidad de tener deteriorada la capacidad cognitiva de una persona mayor, ajustada por las variables analizadas, muestra algunos cambios indicando que se pudo haber sobreestimado el riesgo cuando se calcularon las medidas crudas. Con respecto a la edad, los mayores de 75 años conservan su mayor probabilidad después de ajustar por las otras variables

Teniendo en cuenta la residencia, luego de ajustar, continúa evidenciándose que la región de mayor riesgo para el deterioro cognitivo es la de Magdalena Medio y Medellín, sin significancia estadística, pero que vivir en las subregiones de Bajo Cauca, Occidente y Urabá protege de padecer el evento. En cuanto al estado conyugal, el mayor riesgo se presenta en los adultos mayores sin pareja, aunque estos valores no fueron significativos.

Con respecto a la escolaridad se pudo evidenciar que no contar con algún grado de escolaridad se considera como factor que aumenta el deterioro cognitivo (Tabla 3), siendo esta la característica que presentó la mayor fuerza de asociación ( $R P=8,04$ [5,555$11,630])$ aun después de controlarse por otras variables. También conservó su asociación no participar en actividades o grupos comunitarios y estar afiliado al régimen subsidiado del sistema general de seguridad social en salud.

La mala percepción de calidad de vida se encontraba asociada al riesgo de deterioro cognitivo y, sin embargo, después de ajustar los valores de las RP de los resultados, estos perdieron su significancia estadística. Los adultos mayores insatisfechos con su estado de salud presentaban también mayor riesgo de deterioro cognitivo, situación que se mantiene luego de ajustar por las demás variables, estos valores se consideran significativos. 
Cardona, A.S. et al. Riesgo de deterioro cognitivo en personas mayores de las subregiones de Antioquia, Colombia

TABLA 3

Estimación del RP crudo y ajustado para las variables asociadas con el riesgo de deterioro cognitivo Departamento de Antioquia - 2012

\begin{tabular}{|c|c|c|c|c|c|c|}
\hline \multirow{2}{*}{ Variable } & \multirow{2}{*}{ RP crudo } & \multicolumn{2}{|c|}{ IC $95 \%$} & \multirow{2}{*}{$\begin{array}{c}\mathrm{RP} \\
\text { ajustado }\end{array}$} & \multicolumn{2}{|c|}{ IC $95 \%$} \\
\hline & & Inferior & Superior & & Inferior & Superior \\
\hline \multicolumn{7}{|l|}{ Subregión } \\
\hline Bajo Cauca & 0,77 & 0,544 & 1,078 & $0,40^{*}$ & 0,268 & 0,605 \\
\hline Magdalena Medio & 2,44 & 1,597 & 3,736 & 1,44 & 0,900 & 2,304 \\
\hline Medellín & 1,05 & 0,739 & 1,488 & 1,22 & 0,805 & 1,836 \\
\hline Nordeste & 1,08 & 0,756 & 1,556 & 0,87 & 0,568 & 1,323 \\
\hline Norte & 1,38 & 0,940 & 2,025 & 0,64 & 0,390 & 1,065 \\
\hline Occidente & 0,85 & 0,595 & 1,206 & $0,57^{\star}$ & 0,375 & 0,868 \\
\hline Oriente & 1,21 & 0,828 & 1,750 & 1,33 & 0,857 & 2,077 \\
\hline Suroeste & 1,15 & 0,797 & 1,656 & 0,94 & 0,615 & 1,425 \\
\hline Urabá & 1,00 & 0,701 & 1,436 & $0,55^{\star}$ & 0,349 & 0,852 \\
\hline Valle de Aburrá & 1,00 & - & - & 1,00 & - & - \\
\hline \multicolumn{7}{|l|}{ Persona mayor } \\
\hline 60 a 74 años & 1,00 & - & - & 1,00 & - & - \\
\hline $75+$ años & 2,68 & 2,161 & 3,315 & $2,16^{\star}$ & 1,687 & 2,752 \\
\hline \multicolumn{7}{|l|}{ Estado conyugal } \\
\hline Sin pareja & 1,40 & 1,184 & 1,644 & 1,14 & 0,947 & 1,377 \\
\hline Con pareja & 1,00 & - & - & 1,00 & - & - \\
\hline \multicolumn{7}{|l|}{ Escolaridad } \\
\hline Sin educación & 9,03 & 6,602 & 12,360 & $8,04^{*}$ & 5,555 & 11,630 \\
\hline Primaria & 2,99 & 2,468 & 3,644 & $2,76^{\star}$ & 2,190 & 3,468 \\
\hline Secundaria & 1,00 & - & - & 1,00 & - & - \\
\hline \multicolumn{7}{|l|}{ Recursos sociales } \\
\hline Buenos & 1,00 & - & - & 1,00 & - & - \\
\hline Deteriorados & 1,61 & 1,334 & 1,941 & 0,99 & 0,795 & 1,238 \\
\hline \multicolumn{7}{|l|}{ Régimen de afiliación } \\
\hline Contributivo & 1,00 & - & - & 1,00 & - & - \\
\hline Subsidiado & 1,79 & 1,510 & 2,119 & $1,29 *$ & 1,042 & 1,610 \\
\hline Vinc/ninguno & 1,97 & 1,281 & 3,029 & 1,23 & 0,725 & 2,095 \\
\hline \multicolumn{7}{|c|}{ Participación en grupos } \\
\hline Participa & 1,00 & - & - & 1,00 & - & - \\
\hline No participa & 1,35 & 1,143 & 1,592 & $1,26^{*}$ & 1,035 & 1,526 \\
\hline \multicolumn{7}{|l|}{ Consumo de cigarrillo } \\
\hline $\mathrm{Si}$ & 1,43 & 1,113 & 1,839 & 1,32 & 0,999 & 1,749 \\
\hline No & 1,00 & - & - & 1,00 & - & - \\
\hline \multicolumn{7}{|l|}{ Consumo de alcohol } \\
\hline $\mathrm{Si}$ & 0,59 & 0,469 & 0,734 & 0,79 & 0,607 & 1,027 \\
\hline No & 1,00 & - & - & 1,00 & - & - \\
\hline \multicolumn{7}{|l|}{ Calidad de vida } \\
\hline Mala & 1,55 & 1,319 & 1,831 & 1,13 & 0,917 & 1,397 \\
\hline Buena & 1,00 & - & - & 1,00 & - & - \\
\hline \multicolumn{7}{|c|}{ Satisfacción con la salud } \\
\hline Insatisfecho & 1,90 & 1,559 & 2,310 & $1,51^{\star}$ & 1,206 & 1,896 \\
\hline Satisfecho & 1,00 & - & - & 1,00 & - & - \\
\hline \multicolumn{7}{|l|}{ Capacidad funcional } \\
\hline Dependencia & 1,61 & 1,266 & 2,037 & 1,31 & 0,936 & 1,815 \\
\hline Independientes & 1,00 & - & - & 1,00 & - & - \\
\hline
\end{tabular}

Fuente: Proyecto Situación de salud y condiciones de vida de los adultos mayores. Departamento de Antioquia, 2012.

*Asociación estadísticamente significativa con p<0,05. 
Al ajustar por todas las variables independientes, se encontró que el riesgo de deterioro cognitivo aumenta según la subregión de residencia, la edad del adulto mayor, no tener escolaridad, no participar en grupos, estar insatisfecho con su estado de salud y la afiliación subsidiada en seguridad social; las demás variables no mostraron asociaciones significativas (Tabla 3).

\section{Discusión}

El deterioro cognitivo en las personas mayores puede generar un aumento de la mortalidad y se asocia a una mayor utilización de los servicios de salud. Por consiguiente, su detección incrementa el diagnóstico de las causas reversibles y es una ayuda para el manejo del paciente y para el apoyo de la familia. El deterioro cognitivo y el envejecimiento son procesos que traen cambios en el transcurso de la vida. Dichos cambios pueden presentarse de manera diferente entre los individuos, como lo que sucede con algunas funciones cognitivas que pueden declinar o pueden incrementarse, lo que depende entre otras cosas de aspectos familiares y condiciones psiquiátricas previas (ASHMAN; MOHS; HARVEY, 1999).

A partir de la escala MMSE de Folstein para determinar el riesgo de deterioro cognitivo, en Antioquia, Colombia, se encontró que el 83,2\% de los adultos mayores relevados presentó algún grado de deterioro, entre los cuales el $46,9 \%$ fue deterioro leve. Esto podría deberse a que el proceso de envejecimiento trae consigo un declive en las funciones mentales superiores, aunque es importante identificar cuándo estas variaciones podrían considerase patológicas para proceder a una intervención oportuna que retrase el proceso de deterioro. De acuerdo con Lee et al. (2001), la mayoría de las personas mayores sufre una declinación en la función cognitiva que puede deberse a factores de riesgo relacionados con la edad, tales como la demencia y las enfermedades cardiovasculares, y algunas otras se deben al propio proceso de envejecimiento. Existe evidencia de la dificultad para reconocer los trastornos cognitivos entre los adultos mayores (DE PAULO; FOLSTEIN, 1978).

En Estados Unidos, la prevalencia en personas de 65 años y más es de 7,76\% a 16,5\%, muy superior a la encontrada en Antioquia (FOLSTEIN; FOLSTEIN; HUGH, 1975). En La Habana, Cuba, se presentaron prevalencias de aproximadamente 4,2\% en personas de 60 a 64 años, de $12,9 \%$ en adultos de 65 a 79 años y de un $27,0 \%$ en adultos de 80 años o más (PÉREZ et al., 1993), y en Latinoamérica se han reportado tasas de prevalencia de demencia que oscilan entre 3\% y 6\% (KETZOIAN et al., 1997; HERRERA et al., 1997). Gómez et al. (2003) encontraron en la Habana, Cuba, un 33,3\% de deterioro en pacientes con edades entre 85 y 89 años, y del $25,9 \%$ en el grupo de 80 a 84 años. En Europa, el riesgo de deterioro fue del $0,8 \%$ en mayores de 65 a 69 años y del $28,5 \%$ en los mayores de noventa (LOBO et al., 2000).

Esta investigación encontró un mayor riesgo de deterioro en las mujeres, similar a los hallazgos de Copelan et al. (1992), Morgan y Lilley (1993), Gómez et al. (2003) y León et al. 
(2009), pero son pocos los estudios que muestren la relación con el lugar de residencia. En el presente estudio se evidenció que las personas mayores residentes en las subregiones de Antioquia como Magdalena Medio y Valle de Aburrá tienen más riesgo de presentar este deterioro, situación posiblemente explicada por las condiciones socioeconómicas de los habitantes de estas regiones, pero también se ha evidenciado que variables del contexto pueden estar asociadas a la variabilidad de otros trastornos mentales en los adultos mentales como el riesgo de depresión (CARDONA et al., 2015). No se encontraron diferencias por procedencia urbana o rural, como sí las había en México, donde el daño al ADN fue significativamente mayor en el grupo de adultos mayores con deterioro cognitivo del área urbana $(41,7 \%)$ en comparación con los del área rural (22,2\%) (SIRIA; SÁNCHEZ; MENDOZA, 2009).

En cuanto al estado conyugal, sin asociación significativa se identificó mayor probabilidad de padecerlo en quienes estaban viudos, similar a lo encontrado por Mejía et al. (2007), donde la mayor proporción se registró en los adultos mayores casados y viudos. Hernández, Pedraza y Castillo (2008) afirman que los adultos mayores que viven con su pareja obtienen mejores resultados que los que los que conviven con parientes o solos, tal vez porque estos pacientes tienen mayor autonomía funcional, que está relacionada con capacidades cognitivas mayormente conservadas.

La prevalencia del deterioro cognoscitivo es mayor en sujetos con bajo logro educativo (MEJÍA et al., 2007), las personas educadas tienen mayor capacidad para enfrentar el deterioro ocasionado por la perdida neuronal que llega con el envejecimiento (KATZMAN, 1993). Al analizar los resultados se observó que contar con algún grado de escolaridad es un factor que reduce el riesgo de deterioro cognitivo. Este resultado es similar a los hallazgos del estudio de Gómez et al. (2003), en el que el deterioro fue menor en personas con mayor educación formal, ya que, en las personas con mayor educación hay mayor desarrollo cerebral (LEÓN et al., 2009).

El consumo de tabaco y alcohol no se encontró asociado al deterioro cognitivo en la presente investigación, hallazgo similar al de Flatiglioni y Wnag (2000) en una investigación en Estados Unidos donde se evaluó la asociación entre el consumo de cigarrillo y la demencia tipo Alzheimer. Sin embargo, existen estudios en los cuales sí se ha encontrado algún tipo de asociación (GÓMEZ et al., 2003).

Son pocas las publicaciones que relacionan el riesgo de deterioro con la percepción de la calidad de vida y en la mayoría de los casos solo asocian algunas características principalmente de tipo demográfico y social. Hernández, Pedraza y Castillo (2008) encontraron que la percepción de mala calidad de vida se asocia al riesgo de deterioro cognitivo. La importancia del mantenimiento de las capacidades personales en el adulto mayor y por lo tanto la conservación de la función física y cognitiva ayudan al mantenimiento de una vida con calidad. Un estudio realizado en México considera que no debe evaluarse la calidad de vida en pacientes con algún tipo de demencia, ya que se "pierde la capacidad de evaluar subjetivamente su bienestar, de evaluar su propia calidad de vida o de comunicar a los demás esta valoración” (GARCíA et al., 2003, p. 536). 
Como ya se adelantó en la sección Resultados, en este estudio no se encontró asociación estadística entre el riesgo de deterioro cognitivo con la capacidad funcional de la persona mayor, pero sí se aumenta la probabilidad de padecerlo cuando se aumenta la severidad en las limitaciones para realizar actividades básicas como bañarse o vestirse, entre otras; hallazgos estos similares a los de Cuba según Gómez et al. (2003), Pérez et al. (1993) y Gómez y Rivero (1998), quienes consideraron que un deterioro de origen vascular contribuye a limitar la capacidad funcional.

En diferentes estudios en los que también se empleó el MMSE en personas mayores, se encontró que la función mental más afectada es la memoria de evocación (GÓMEZ et al., 2003). En la presente investigación la puntación para la memoria de evocación fue para un poco más de la mitad de los adultos mayores (56\%) -coincidiendo con otros estudios-, pero se recomienda tener cuidado al momento de interpretarla (MARCOS, 1991). Las diferencias entre los resultados de las investigaciones revisadas y los hallazgos del presente estudio pueden deberse a las principales limitaciones que se tuvieron, como las características culturales de las poblaciones investigadas, que afectan directamente su conducta, el instrumento de medición sin la aplicación de otros instrumentos de carácter psicométrico y por ser un estudio descriptivo transversal, sin posibilidad de mirar causalidad. Adicionalmente, se debe considerar que las capacidades mentales superiores de las personas mayores sufren un deterioro propio de la edad, visto que con el envejecimiento las redes neuronales empiezan a debilitarse por la desmielinización del sistema nervioso, lo cual hace que el rendimiento de la persona no sea el mismo en todas las pruebas (PÉREZ; GARCÍA, 2006).

\section{Conclusiones}

El 83,1\% de los adultos mayores presenta algún riesgo de deterioro, principalmente leve (46,8\%), y solo el $16,8 \%$ no tiene este riesgo. Según el modelo estadístico calculado, se encontró evidencia estadísticamente significativa de que la edad más adulta, vivir sin pareja - con predominio de la viudez-, no tener ninguna escolaridad o solo primaria, estar afiliado en el régimen subsidiado de la seguridad social en salud, no participar en grupos comunitarios, percibir la calidad de vida como mala, estar insatisfecho con el estado de salud y la dependencia física para realizar actividades cotidianas se encontraron asociados con la prevalencia de riesgo de deterioro cognitivo.

Los resultados mencionados anteriormente permiten orientar los programas de salud pública de Antioquia y Colombia, dirigidos a reducir el riesgo de este padecimiento, que está asociado a la edad, pero puede disminuirse con la inclusión de las personas mayores en programas educativos, la participación en grupos comunitarios, la tamización constante en los servicios de salud, el fortalecimiento de los recursos sociales y el fomento de la actividad física en la vejez, para lograr un envejecimiento saludable con mejor calidad de vida. 


\section{Conflicto de intereses}

Se deja constancia de que no existió ningún conflicto entre las partes involucradas ni con los adultos mayores de Antioquia.

\section{Referencias}

AGRIGOROAEI, S.; LACHMAN, M. Cognitive functioning in midlife and old age: combined effects of psychosocial and behavioral factors. The Journals of Gerontology Series B: Psychological Sciences and Social Sciences. v. 66B, Supplement 1, p. 130-140, 2011.

ASHMAN, T.; MOHS, R.; HARVEY, P. Cognition and aging. Principles of geriatric medicine and gerontology. USA: McGraw-Hill, 1999. p. 1219-1228.

BIURRUMUNZUÉ, A. Impacto de la enfermedad de Alzheimer en la familia. Geriátrica. v. 16, n. 4, p. 45-95, 2000.

CARDONA, D.; SEGURA, A. M.; GARZÓN, M. O. Situación de salud y condiciones de vida del adulto mayor. Departamento de Antioquia, 2012. Medellín: Universidad CES/Gobernación de Antioquia, 2013.

Efectos contextuales asociados a la variabilidad del riesgo de depresión de los adultos mayores. Departamento de Antioquia, 2012. Biomédica, v. 35, n. 1, p. 73-80, 2015.

CONSEJO DE ORGANIZACIONES INTERNACIONALES DE LAS CIENCIAS MÉDICAS; ORGANIZACIÓN MUNDIAL DE LA SALUD. Pautas éticas internacionales para la investigación biomédica en seres humanos. Ginebra: CIOMS/OMS, 2002.

COPELAN, J.; DAVISON, A.; DEWEY, M. E. Pseudo-dementia: prevalence, incidence and three-years outcome, in Liverpool. British Journal of Psychiatry, v. 161, p. 230-239, 1992.

CRICHTON, R. Some problems of clinical trials in elderly people. Archives of neurology, v. 3, p. 247-253, 1961.

DE PAULO, J. R.; FOLSTEIN, M. F. Psychiatric disturbance in neurological patients. Detection, recognition and hospital course. Annals of Neurology, v. 4, p. 225-228, 1978.

DANE - Departamento Administrativo Nacional De Estadística. Proyecciones de población: proyecciones municipales 2005-2011. 2007. Disponible en: 〈http://dane.gov.co〉. Acceso en: 10 abr. 2015.

FEDERACIÓN MUNDIAL DE SALUD MENTAL. La salud mental y el envejecimiento. Psiquiatría Pública, v. 11, n. 5-6, p. 169-175, 1999.

FLATIGLIONI, L.; WNAG, H.X. Smoking and Parkinson's disease and Alzheimer's disease: review of the epidemiological studies. Behavioural Brain Research, v. 113, n. 1-2, p. 117-120, 2000.

FOLSTEIN, M.; FOLSTEIN, S.; HUGH, P. R. Minimental state: a practical method for grading the cognitive state of the for clinician. Journal of Psychiatric Research, v. 12, p. 189-198, 1975.

FRIEDLAND, R. P. Epidemiology, education and ecology of Alzheimer's disease. Neurology, v. 43, p. 246-249, 1993.

GARCÍA, M.; SÁNCHEZ, M.; LIEBANA, A.; PÉREZ, V.; PÉREZ, P. F.; VIEDMA, G.; BORREGO, J.; GIL, J. Calidad de vida relacionada con la salud en pacientes ancianos en hemodiálisis. Nefrología, v. 23, n. 6, p. 528-537, 2003. 
GOBERNACIÓN DE ANTIOQUIA. Adulto mayor. Disponible en: 〈http://www.antioquia.gov.co/index. php/62-plan-de-desarrollo/linea-estrategica-2/2512-adulto-mayor . Acceso en: 4 sep. 2015.

. Regiones de Antioquia. Disponible en: <http://antioquia.gov.co/index.php/antioquia/ datos-de-antioquia/9790\#>. Acceso en: 4 sep. 2015.

GOLDBERG, D. Manual del general health questionnaire. Windsor: NFER Publishing, 1978.

GOLDBERG, D.; HILLER, V. A scaled version of the general health questionnaire. Psychological Medicine, v. 1, p. 139-45, 1979.

GÓMEZ, N.; BONNIN, B.; GÓMEZ, M.; YÁNEZ, B.; GÓNZALEZ, A. Caracterización clínica de los pacientes con deterioro cognitivo. Revista Cubana de Medicina, v. 42, n. 1, p. 12-17, 2003.

GÓMEZ, N.; RIVERO, E. Características clínicas e imagenológicas de la demencia vascular. Revista Cubana de Medicina, v. 37, n. 1, p. 6-12, 1998.

HERNÁNDEZ, E.; PEDRAZA, G.; CASTILLO, M. Deterioro cognitivo y calidad de vida en ancianos de una clínica de medicina familiar de la ciudad de México. Archivos en Medicina Familiar, v. 10, n. 4, p. 127-132, 2008.

HERRERA, E.; CARAMELLI, P.; SILVEIRA, A. S.; MATHIAS, S. C.; NITRINI, R. Population epidemiology survey of dementia in Catanduva, Brazil. Preliminary results. Journal of the Neurological Sciences, v. 150, p. 155-156, 1997.

IBARRA, L.; VILLARREAL, J.; SALINAS, R. Capacidad cognitiva de los pacientes de un servicio geriátrico ambulatorio en Monterrey, México. Medicina Universitaria, v. 8, n. 31, p. 84-87, 2006.

KATZ, S.; FORD, A.; MOSKOWITZ, R.; JACKSON, B. A.; JAFFE, M. W. Studies of illness in the age: the index of ADL, a standarized measure of biological and psychosocial function. JAMA, v. 185, p. 914-919, 1963.

KATZMAN, R. Education and the prevalence of dementia and Alzheirmer's disease. Neurology, v. 43, p. 13-20, 1993.

KETZOIAN, C.; ROMERO, S.; DIEGUEZ, E.; CAIROLO, G.; REGA, Y.; CASERES, R. Prevalence of demential sindromes in a population of Uruguay. Study of "Villa del Cerro". Journal of the Neurological Sciences, p. 150-155, 1997.

LEE, L.; KANG, S. A.; LEE, H. O.; LEE, B. H.; PARK, J. S.; KIM, J. H.; JUNG, I. K.; PARK, Y. J.; LEE, J. E. Relationships between dietary intake and cognitive function level in Korean elderly people. Public Health, v. 115, p. 133-138, 2001.

LEÓN, B.; MILIÁN, F.; CAMACHO, N.; ARÉVALO, R. E.; ESCARTíN, M. Factores de riesgo para deterioro cognitivo y funcional en el adulto mayor. Revista Médica del Instituto Mexicano del Seguro Social, v. 47, n. 3, p. 277-284, 2009.

LETURIA, F.; YANGUAS, J.; ARRIOLA, E.; URIARTE, A. Escala de Recursos Sociales (OARS). Duke University Center (1978). La valoración de las personas mayores: evaluar para conocer, conocer para intervenir. Manual práctico. Barcelona: Caritas Española, 2001. p. 313-315.

LOBO, A.; LAUNER, L. J.; FRATIGLIONI, L.; ANDERSON, K.; DI CARLO, A.; BRETELER, M. M. Prevalence of dementia and mayor subtypes in Europe: a collaborative study of population-based cohorts Neurology Disease in the Elderly Research Group. Neurology, v. 54, n. 11, suppl. 5, p. 4-9, 2000.

MARCOS, T. Métodos paraclínicos de diagnóstico en psiquiatría (III): tests neuropsicológicos. Deterioro: concepto y medida. In: VALLEJO, J.; BALLUS, C. Introducción a la psicopatología y la psiquiatría. 3. ed. Barcelona: Salvat, 1991.

MEJÍA, S.; MIGUEL, A.; VILLA, A.; RUIZ, L.; GUTIÉRREZ, L. M. Deterioro cognoscitivo y factores asociados en adultos mayores en México. Salud Publica de Mexico, v. 49, n. 4, p. 475-481, 2007. 
MORGAN, K.; LILLEY, J. M. Indice of dementia in a representative Brit sample. British Journal of Psychiatry, v. 163, p. 467-70, 1993.

ORGANIZACIÓN MUNDIAL DE LA SALUD. Programa Envejecimiento y Ciclo vital. Envejecimiento activo: un marco político. Revista Española de Geriatría y Gerontología, v. 37, n. 74, p. 105, 2002.

PÉREZ, M. D.; GARCÍA, N. Estimulación cognitiva en el anciano dependiente hospitalizado. Gerokomos, v. 17, n. 3, p. 125-131, 2006.

PÉREZ, O.; CASTILLO, V. L.; RAITERI, J.; ROSELLÓ, H.; PÉREZ, J.; GÓMEZ, N. Prevalencia de demencia en la población geriátrica de la Habana Vieja. Revista Española de Neurología, v. 8, n. 1, p. 18-22, 1993.

PÉREZ, V. El deterioro cognitivo: una mirada provisoria. Revista Cubana de Medicina General Integral, v. 21, 2005.

SANCHÉZ, I. Y.; PERÉZ, V. T. El funcionamiento cognitivo en la vejez: atención y percepción en el adulto mayor. Revista Cubana de Medicina General Integral, v. 24, n. 2, p. 1-7, 2008.

SIRIA, J.; SÁNCHEZ, M.; MENDOZA, V. M. Estrés oxidativo y lugar de residencia urbano como factores de riesgo para deterioro cognitivo en adultos mayores. Bioquímica, v. 34, n. 1, p. 131145, 2009.

VIVAS, L.; ANDRÉS, M. L.; CANET, L. Funcionamiento cognitivo y emocional en la vejez: claves para una intervención. 2011. Disponible en: 〈http://www.aggmdp.org.ar/Funcionamiento\%20 cognitivo\%20en\%20la\%20vejez.pdf〉. Acceso en: 10 abr. 2015.

\section{Sobre las autoras}

Angela Segura Cardona es doctora en Epidemiología de la Universidad de Antioquia. Docente investigadora en la Universidad CES, Colombia. Grupo de Investigación Epidemiología y Bioestadística.

Maria Garzón Duque es magíster en Epidemiología de la Universidad de Antioquia. Docente investigadora en la Universidad CES, Colombia. Grupo de Investigación Observatorio de la Salud Pública

Doris Cardona Arango es doctora en Demografía de la Universidad Nacional de Córdoba, Argentina. Docente investigadora en la Universidad CES, Colombia. Grupo de Investigación Epidemiología y Bioestadística.

Alejandra Segura Cardona es magíster en Epidemiología de la Universidad CES. Docente investigadora en la Universidad CES, Facultad de Psicología.

\section{Dirección para correspondencia (todas las autoras)}

Universidad CES

Calle 10 A No. 22-04

050021 - Medellín, Antioquia, Colombia

\section{Resumo}

Risco de comprometimento cognitivo em pessoas idosas nas sub-regiões de Antioquia, Colômbia

Neste artigo, procura-se determinar a prevalência de risco de comprometimento cognitivo, a partir da escala minimental, e sua relação com fatores demográficos, sociais e funcionais em pessoas 
de 60 anos e mais residentes nas sub-regiões de Antioquia, Colômbia. 0 estudo é quantitativo, analítico transversal, com fonte primária de informação e amostra probabilística. Verificouse que $83,1 \%$ dos idosos têm algum risco de comprometimento cognitivo, especialmente mulheres, e está associado a residir nas sub-regiões de Magdalena Medio e Medellín, ter idade mais avançada, viuvez, não ter escolaridade, recursos sociais escassos, não participar em grupos comunitários, má percepção da qualidade de vida, não estar satisfeito com a saúde e dependência funcional para atividades básicas. Estes resultados permitem direcionar ações de saúde pública relacionadas com a inclusão dos idosos em programas educacionais, participação em grupos comunitários, triagem constante nos serviços de saúde, reforço dos recursos sociais e promoção da atividade física na velhice, para o envelhecimento saudável, com melhor qualidade de vida.

Palavras-chave: Idoso. Cognição. Envelhecimento.

\section{Abstract}

Risk of cognitive impairment in elderly people in the subregions of Antioquia, Colombia

We sought to determine the prevalence of risk of cognitive impairment and its relation to demographic, social and functional factors, in the older population of the subregions of Antioquia, Colombia. The study is quantitative, cross-sectional analytical, utilizing primary source of information and probabilistic sample. The risk of cognitive impairment was determined by using the minimental scale. The results show that $83.1 \%$ of the elderly have some risk, especially women, and is statistically associated with living in the sub-region of Magdalena Medio and Medellin, being older, widowhood, not having schooling, having scarce social resources, do not participate in community groups, have poor perception of quality of life, and to be dissatisfied with health and functional dependence for basic activities. These results allow for targeting public health programs related to the inclusion of elderly in educational programs, participation in community groups, constant screening in health services, strengthening of social resources and promoting physical activity at older age, for a healthy aging with a better quality of life.

Keyword: Elderly. Cognition. Aging.

Recebido para publicação em 21/06/2016 Recomendado para publicação em 17/11/2016 Aceito para publicação em 22/12/2016 